\title{
COMPLETELY BOUNDED ISOMORPHISMS OF INJECTIVE VON NEUMANN ALGEBRAS
}

\author{
by ERIK CHRISTENSEN and ALLAN M. SINCLAIR
}

(Received 31st March 1988)

Milutin's Theorem states that if $X$ and $Y$ are uncountable metrizable compact Hausdorff spaces, then $C(X)$ and $C(Y)$ are isomorphic as Banach spaces [15, p. 379]. Thus there is only one isomorphism class of such Banach spaces. There is also an extensive theory of the Banach-Mazur distance between various classes of classical Banach spaces with the deepest results depending on probabilistic and asymptotic estimates [18]. Lindenstrauss, Haagerup and possibly others know that as Banach spaces

$$
B(H) \approx \bigoplus_{1}^{\infty} M_{n}(\mathbb{C}) \approx R,
$$

where $H$ is the infinite dimensional separable Hilbert space, $R$ is the injective $\mathrm{II}_{1}$-factor on $H$, and $\approx$ denotes Banach space isomorphism. Haagerup informed us of this result, and suggested considering completely bounded isomorphisms; it is a pleasure to acknowledge his suggestion. We replace Banach space isomorphisms by completely bounded isomorphisms that preserve the linear structure and involution, but not the product. One of the two theorems of this paper is a strengthened version of the above result: if $N$ is an injective von Neumann algebra with separable predual and not finite type I of bounded degree, then $N$ is completely boundedly isomorphic to $B(H)$. The methods used are similar to those in Banach space theory with complete boundedness needing a little care at various points in the argument. Extensive use is made of the conditional expectation available for injective algebras, and the methods do not apply to the interesting problems of completely bounded isomorphisms of non-injective von Neumann algebras (see [4] for a study of the completely bounded approximation property). The trace preserving normal conditional expectation available for type $\mathrm{II}_{1}$ von Neumann algebras is exploited to obtain the second Theorem (1.2): if $M$ and $N$ are type $\mathrm{II}_{1}$ von Neumann algebras with $M \hookrightarrow N, N \hookrightarrow M$, and $M \oplus M \hookrightarrow M$, then $M$ and $N$ are completely boundedly normally isomorphic. Here $\hookrightarrow$ denotes a normal *-monomorphism from the one von Neumann algebra into another that is not necessarily unital. Our results are not directly related to those on perturbation in von Neumann 
algebras $[1,2]$ as the distance between the algebras used here is different from that used in perturbation theory.

The basic definitions and the main results of this paper (Theorems 1.1 and 1.2) are given below. In Section 2 we prove 1.1 giving a detailed account even when the lemmas are only modifications of the Banach space results, in the hope of making this paper easier to read. Theorem 1.2 and a couple of corollaries are proved in Section 3. There is brief further discussion at the end of Section 2.

The standard theory of completely bounded linear operators between $C^{*}$-algebras is carefully expounded in Paulsen's lecture notes [12]. Let $M_{n}(\mathbb{C})$, or $M_{n}$, denote the algebra of $n \times n$ matrices over $\mathbb{C}$, and let $\otimes$ denote the spacial tensor product of $C^{*}$. algebras. An operator space $X$ is a subspace of $B(H)$, and associated with it is the norm on $M_{n}(X)$ inherited from $M_{n}(B(H))=M_{n}(\mathbb{C}) \otimes B(H)$ for all $n \in \mathbb{N}$. If $\phi$ is a linear operator from one operator space $X$ into another $Y$, define $\phi_{n}: M_{n}(X) \rightarrow M_{n}(Y)$ by $\phi_{n}=i_{n} \otimes \phi$, where $i_{n}$ is the identity operator on $M_{n}(\mathbb{C})$. The operator $\phi$ is completely bounded if $\|\phi\|_{c b}=\sup \left\{\left\|\phi_{n}\right\|: n \in \mathbb{N}\right\}$ is finite. Many of the completely bounded operators we use below will be constructed from the characterisation of completely bounded operators in terms of a representation and two bridging operators (see [12] for a detailed discussion).

Let $X \subseteq B(H)$ be an operator space and let $\phi: X \rightarrow B(K)$ be a linear operator, where $H$ and $K$ are Hilbert spaces. Then $\phi$ is completely bounded if, and only if, there is a *-representation $\pi$ of $B(H)$ on a Hilbert space $H_{\pi}$ and continuous linear operators $U: H_{\pi} \rightarrow K$ and $V: K \rightarrow H_{\pi}$ such that $\phi(x)=U \pi(x) V$ for all $x \in X$; further $U, V$, and $\pi$ may be chosen so that $\|\phi\|_{c b}=\|U\| \cdot\|V\|$. If $\phi$ is normal, then $\pi$ may be chosen to be normal.

Note that the completely bounded norm satisfies the standard inequalities $\|\phi+\psi\|_{c b} \leqq$ $\|\phi\|_{c b}+\|\psi\|_{c b}$ and $\|\phi \circ \psi\|_{c b} \leqq\|\phi\|_{c b} \cdot\|\psi\|_{c b}$. Throughout $\oplus$ will denote the direct sum with $l_{\infty}$ norm; observe that it is a direct sum of operator spaces because it induces a natural direct sum on $B(H)$ and $M_{n}(B(H) \oplus B(H))=M_{n}(B(H)) \oplus M_{n}(B(H))$ [13].

If $X$ and $Y$ are operator spaces, $X$ and $Y$ are said to be completely boundedly isomorphic, written $X c b \cong Y$, if there is a linear isomorphism $\phi$ (probably not preserving the product structure) such that $\phi$ and $\phi^{-1}$ are completely bounded. Let

$$
d c b(X, Y)=\inf \left\{\|\phi\|_{c b} \cdot\left\|\phi^{-1}\right\|_{c b}: \phi: X \rightarrow Y \text { with } \phi \text { and } \phi^{-1} \text { completely bounded }\right\}
$$

be the completely bounded distance between $X$ and $Y$. If $\phi$ and $\phi^{-1}$ are normal, we write $X \sigma c b \cong Y$ and let $d \sigma c b(X, Y)$ be the corresponding normal distance between $X$ and $Y$. An operator $\phi$ between two $C^{*}$-algebras is said to preserve the involution if $\phi\left(x^{*}\right)=\phi(x)^{*}$ for all $x$. Throughout the isomorphisms will be taken to be involution preserving: the exception is the maps constructed in Lemma 2.6; however, modifying that operator leads to an involution preserving completely bounded isomorphism.

Theorem 1.1 If $N$ is an injective von Neumann algebra with separable predual and not finite type $I$ of bounded degree, then $N$ is completely boundedly isomorphic to $B(H)$ by an involution preserving operator. Further $d c b(N, B(H)) \leqq 2^{16}$.

The hypothesis of separable predual is to ensure $N$ has a faithful normal represen- 
tation on $H$. Finite type I algebras of bounded degree are completely boundedly isomorphic to commutative algebras so cannot be Banach space isomorphic to $B(H)$. Corollary 2.7 lists the isomorphism classes of injective von Neumann algebras with separable preduals: $l_{\infty}^{n}(n \in \mathbb{N}), l_{\infty}, B(H)$. Theorem 1.1 may be used to transfer operator space or Banach space information from $B(H)$ to the von Neumann algebra $N$; for example, the failure of the approximation property. (Corollary 2.14.)

With care in the calculations and minor changes to the proof one can show that if the von Neumann algebra $N$ in Theorem 1.1 has no finite type I central direct summand, then the completely bounded isomorphism may be chosen to preserve the identity.

Theorem 1.2. If $M$ and $N$ are type $I_{1}$ von Neumann algebras with $M \hookrightarrow N, N \hookrightarrow M$, and $M \oplus M \hookrightarrow M$, then $M$ and $N$ are completely boundedly normally isomorphic. Further $d \sigma c b(M, N) \leqq 2^{16}$.

The above result is a weak type of Schröder-Bernstein Theorem but with the initial injections preserving the algebra structure and the final isomorphism not doing so. The hypothesis $M \oplus M \hookrightarrow M$ is implied by $M \cong M \bar{\otimes} R$, where $R$ is the unique injective $\mathrm{II}_{1}$ factor. Can the condition $M \oplus M \hookrightarrow M$ be dropped in the above hypotheses? This problem is related to the example von Neumann gives in the appendix of the fourth "On rings of operators" paper, where he constructs two type $\mathrm{II}_{1}$ factors $M$ and $N$ with $M \hookrightarrow N$ and $N \hookrightarrow M$ but $M$ not isomorphic to $N$.

We wish to thank Guyan Robertson for drawing Lemma 2.2 to our notice after the first version of this paper was complete; his suggestions simplified the proof of Theorem 1.1 .

\section{Injective algebras}

In general hypotheses involving complete positivity are considerably stronger than those involving complete boundedness. Replacing the hypothesis of complete boundedness of $\phi$ and $\phi^{-1}$ by their complete positivity for unital $C^{*}$-algebras easily leads to the algebras being isomorphic. The proof involves standard techniques; see [12] for more about complete positivity and isomorphisms.

Lemma 2.1. Let $A$ and $B$ be unital $C^{*}$-algebras, and let $\phi$ be a linear isomorphism from $A$ onto $B$. If $\phi$ and $\phi^{-1}$ are completely positive, then $A$ is *-isomorphic to $B$ by the *-isomorphism

$$
\theta(a)=\phi(1)^{-1 / 2} \phi(a) \phi(1)^{-1 / 2} \quad(a \in A)
$$

Proof. Stinespring's Theorem ensures that there is a representation of $\phi$ as $\phi(a)=V^{*} \pi(a) V$, where $\pi$ is a $*$-representation of $A$ on a Hilbert space $K$, and $V: H \rightarrow K$ is a continuous linear operator where $B \subseteq B(H)$. Then $\left\|\phi^{-1}\right\| V^{*} V \geqq V^{*} \pi\left(\phi^{-1}(1)\right) V=$ $\phi\left(\phi^{-1}(1)\right)=1$ so that $V^{*} V=\phi(1)$ is a positive invertible element of $B$. Hence $\theta$ is well 
defined, and $\theta$ and $\theta^{-1}$ are completely positive linear isomorphisms from $A$ onto $B$, and $B$ onto $A$, with $\theta(1)=1$. Hence $\|\theta\|_{c b}=\left\|\theta^{-1}\right\|_{c b}=1$ and $\theta$ is a complete isometry. Thus $\theta_{2}$ is a Jordan isomorphism from $M_{2}(A)$ onto $M_{2}(B)$ as it is a unital isometry between these $C^{*}$-algebras [8]. Applying $\theta_{2}$ to the Jordan product

$$
\left[\begin{array}{ll}
x & 0 \\
0 & 0
\end{array}\right] \circ\left[\begin{array}{ll}
0 & y \\
0 & 0
\end{array}\right]
$$

shows that $\theta$ is a homomorphism, which completes the proof.

The referee has kindly pointed out that the above lemma is true with completely positive weakened to 2-positive with the use of Stinespring replaced by

$$
\left\|\phi^{-1}\right\| \phi(1)=\phi\left(\left\|\phi^{-1}\right\| 1\right) \geqq \phi\left(\phi^{-1}(1)\right)=1 .
$$

Before embarking on the proof of the various lemmas we record a few elementary facts about completely bounded isomorphisms.

If $E$ is a completely bounded projection on a von Neumann algebra $N$ with $\|E\|_{c b}=1$, then $N c b \cong E N \oplus(I-E) N$ and $d c b(N, E N \oplus(I-E) N) \leqq 4$. This is trivial using $\psi: N \rightarrow$ $E N \oplus(I-E) N: x \mapsto E x \oplus(I-E) x$ with $\|\psi\|_{c b} \leqq 2$ and $\left\|\psi^{-1}\right\|_{c b} \leqq 2$.

If $A c b \cong B$ and $M c b \cong N$, then $A \oplus M c b \cong B \oplus N$ and $d c b(A \oplus M, B \oplus N) \leqq$ $\max (d c b(A, B), d c b(M, N))$.

Finally

$$
d c b(X, Z) \leqq d c b(X, Y) \cdot d c b(Y, Z)
$$

Corresponding results hold for normal completely bounded isomorphisms both here and in the lemma below. If $X$ is an operator space, let $X^{\infty}$ denote the operator space obtained by taking the $l^{\infty}$ direct sum of countably infinitely many copies of $X$.

The main proof depends on a Banach space argument using a lemma of Pelczynski's to obtain the Schröder-Bernstein result required [10, 2.a.3, p. 54].

Lemma 2.2. If $B, N, X, Y$ are operator spaces with $B c b \cong B^{\infty}, B c b \cong N \oplus X$ and $N c b \cong B \oplus Y$ then $B c b \cong N$ and $d c b(B, N) \leqq \alpha^{2} \beta^{2} \gamma^{2}$, where $\alpha, \beta, \gamma$ are the completely bounded distances in the three isomorphisms, respectively.

Proof. This is a standard algebraic calculation using the result that the permutation on a direct sum is a complete isometry:

$$
\begin{aligned}
B c b & \cong B^{\infty} c b \cong(N \oplus X)^{\infty} \\
c b & \cong N^{\infty} \oplus X^{\infty} c b \cong N^{\infty} \oplus X^{\infty} \oplus N \\
c b & \cong B^{\infty} \oplus N
\end{aligned}
$$




$$
\begin{aligned}
& c b \cong B^{\infty} \oplus B \oplus Y c b \cong B^{\infty} \oplus Y \\
& c b \cong B \oplus Y c b \cong N .
\end{aligned}
$$

The isomorphisms $B c b \cong N \oplus X$ and $N c b \cong B \oplus Y$ in the above lemma will follow from injectivity in 1.1 and from the trace preserving conditional expectation in 1.2.

Lemma 2.3. If $B=B(H)$ is the algebra of all bounded linear operators on a separable infinite dimensional Hilbert space $H$, then $B c b \cong B^{\infty}$ with $d c b\left(B, B^{\infty}\right) \leqq 16$.

Proof. There is a natural normal *-monomorphism from $B^{\infty}$ into $B$ obtained from $I=\sum_{1}^{\infty} p_{j}$ by identifying $0 \oplus \cdots \oplus 0 \oplus B \oplus 0$ with $p_{j} B \mid p_{j} H$, where $p_{j}$ are infinite dimensional projections on $H$ with $p_{j} p_{k}=0$ if $j \neq k$. Thus $B c b \cong B^{\infty} \oplus X$ since there is a completely bounded normal projection from $B$ onto $B^{\infty}$ given by

$$
x \mapsto \oplus\left(p_{j} x \mid p_{j} H\right) .
$$

Hence

$$
\begin{gathered}
B c b \cong B^{\infty} \oplus X c b \cong B^{\infty} \oplus B^{\infty} \oplus X \\
c b \cong B^{\infty} \oplus B c b \cong B^{\infty} .
\end{gathered}
$$

Two of the isomorphisms are complete isometries and the others have distance 4 , so $\operatorname{dcb}\left(B, B^{\infty}\right) \leqq 16$.

The following lemma is essentially in [19].

Lemma 2.4. Let $N$ be an injective von Neumann algebra which is not finite type $I$ of bounded degree. Then there is a normal *-monomorphism from $\mathscr{M}=\bigoplus_{1}^{\infty} M_{n}(\mathbb{C})$ into $N$.

Proof. If $N$ has a central summand which is not finite of type 1 , then inside this summand there are pairwise orthogonal projections $p_{i j}$ for $1 \leqq i<\infty$ and $1 \leqq j \leqq i$ so that $v_{i j}^{*} v_{i j}=p_{i 1}$ and $v_{i j} v_{i j}^{*}=p_{i j}$; this is just the definition of $p_{i 1} \sim p_{i j}$. Then the von Neumann subalgebra of $N$ generated by the set $\left\{v_{i j}: 1 \leqq i<\infty, 1 \leqq j \leqq i\right)$ is isomorphic to $\mathscr{M}$. If $N$ is finite of type I, then

$$
N \cong \bigoplus_{1}^{\infty} M_{m(j)}\left(A_{j}\right)
$$

where $A_{j}$ are abelian von Neumann algebras and $m(j)$ tends to infinity with $j$ [9]. We inductively choose a sequence $j(n)$ in $\mathbb{N}$ such that $j(n+1)>j(n)$ and $j(n) \geqq n$ for all $n$. The algebra $\mathscr{M}$ is now embedded into $N$ by embedding $M_{n}(\mathbb{C})$ into $M_{m(j(n))}\left(A_{j(n)}\right)$ for each $n \in \mathbb{N}$. 
The crucial idea of using a sequence $p_{n}$ in the following proof is known to Linderstrauss and Haagerup.

Proof of Theorem 1.1. The hypotheses of Lemma 2.2 are checked with $B=B(H)$. By hypothesis the von Neumann algebra $N$ has a faithful normal representation on $H$ and there is a completely bounded conditional expectation $E$ from $B(H)$ onto $N$; thus $B(H) c b \cong N \oplus X$, where $X=\operatorname{Ker} E$.

Let $p_{n}$ be an increasing sequence of finite rank projections in $B(H)$ with $\operatorname{dim} p_{n} H=n$ and $p_{n}$ converging strongly to $I$. Let $\phi: B(H) \rightarrow \mathscr{M}=\bigoplus_{1}^{\infty} M_{n}(\mathbb{C})$ be defined by $\phi(x)=$ $\oplus p_{n} x p_{n}$, where $p_{n} B(H) p_{n}$ is identified with $M_{n}(\mathbb{C})$. Then $\phi$ is completely bounded with $\|\phi\|_{c b}=1$. Define $\psi: \mathscr{M} \rightarrow B(H)$ by $\left\langle\psi\left(\left(x_{n}\right)\right) \xi, \eta\right\rangle=\operatorname{LIM}\left\langle\chi_{n}\left(x_{n}\right) \xi, \eta\right\rangle$ where $\chi_{n}$ is the natural map used to identify $M_{n}(\mathbb{C})$ with $p_{n} B(H) p_{n}$ and $\chi_{n+1}\left(y_{n} \oplus 0\right)=\chi_{n}\left(y_{n}\right)$ for all $y_{n} \in M_{n}(\mathbb{C})$ and all $n \in \mathbb{N}$; here LIM is a Banach limit on $\mathbb{N}$. Then $\psi$ is a completely bounded operator from $\mathscr{M}$ into $B(H)$ with $\|\psi\|_{\mathfrak{c b}}=1$. Further $\psi \circ \phi$ is the identity on $B(H)$ and $\phi \circ \psi$ is the projection from $\mathscr{M}$ onto $\phi(B(H)$ ). By Lemma 2.4 there is a normal *-monomorphism from $\mathscr{M}$ into $N$. The injectivity of $\mathscr{M}$ gives a conditional expectation $F$ from $N$ onto $\mathscr{M}$, and the projection $\phi \circ \psi$ carries $\mathscr{M}$ onto $\phi(B(H))$ so $N c b \cong B(H) \oplus Y$, where $Y=\operatorname{Ker} \phi \circ \psi \circ F$.

Lemma 2.3 ensures that the remaining hypothesis of Lemma 2.2 is satisfied, and that $\alpha \leqq 16$. The remark after Lemma 2.1 shows that $\beta \leqq 4$ and $\gamma \leqq 4$ in Lemma 2.2. This gives the estimate in Theorem 1.1 , since $\operatorname{dc} b(N, B) \leqq \alpha^{2} \beta^{2} \gamma^{2}=2^{16}$.

The following elementary lemma is used in the proof of Corollary 2.7. We are grateful to Tomczak-Jaegerman for informing us that the Banach space distance $d\left(M_{n}(\mathbb{C}), \mathbb{C}^{n^{2}}\right)$ between $M_{n}(\mathbb{C})$ and $\mathbb{C}^{n^{2}}$ is greater than $c n$, where $0<c<1$ is a universal constant (see [18] for a detailed discussion and further references). Is $d c b\left(M_{n}(\mathbb{C}), \mathbb{C}^{n^{2}}\right)=n$ ?

Lemma 2.6. Let $A$ be a $C^{*}$-algebra and let $n \in \mathbb{N}$. Then $M_{n}(A) c b \cong A^{n^{2}}$ and $d c b\left(M_{n}(A), A^{n^{2}}\right) \leqq n$.

Proof. This result is just $M_{n}(\mathbb{C}) c b \cong \mathbb{C}^{n^{2}}$ tensored with the identity operator on $A$, since $\|\phi \otimes I\|_{c b}=\|\phi\|_{c b}$ for a completely bounded operator $\phi$ [12]. A linear isomorphism $\phi$ between the finite dimensional $C^{*}$-algebras $M_{n}(\mathbb{C})$ and $\mathbb{C}^{n^{2}}$ is completely bounded so $M_{n}(\mathbb{C}) c b \cong \mathbb{C}^{n^{2}}$.

We obtain the estimate on the completely bounded distance by calculating $\|\phi\|_{c b}$ for an obvious $\phi$. Let

$$
\phi\left(a_{i j}\right)=\left(a_{11}, a_{12}, \ldots, a_{1 n}, a_{21}, \ldots, a_{2 n}, \ldots, a_{n 1}, \ldots, a_{n n}\right)
$$

for all $\left(a_{i j}\right) \in M_{n}(A)$. We shall show that $\|\phi\|_{c b}=1$ and $\left\|\phi^{-1}\right\|_{c b} \leqq n$ by writing down representations of the completely bounded linear operators $\phi$ and $\phi^{-1}$. Let $e_{i j}$ $(1 \leqq i, j \leqq n)$ be the standard matrix units of $M_{n}(\mathbb{C})$, and let $\pi$ be the $n^{2}$-amplification of the natural representation of $M_{n}(\mathbb{C})$ on the $n$-dimensional Hilbert space. Then $\pi(a)=$ $a \otimes I_{n^{2}}$ for all $a \in M_{n}(\mathbb{C})$. Let 


$$
W=\operatorname{diag}\left(e_{11}, \ldots, e_{11}, e_{12}, \ldots, e_{12}, \ldots, e_{1 n}, \ldots, e_{1 n}\right)
$$

with each element $e_{1 j}$ repeated $n$-times, and let

$$
V=\operatorname{diag}\left(e_{11}, e_{21}, \ldots, e_{n 1}, e_{11}, e_{21}, \ldots, e_{n 1}, \ldots, e_{11}, e_{21}, \ldots, e_{n 1}\right)
$$

with each $n$-tuple $\left(e_{11}, e_{21}, \ldots, e_{n 1}\right)$ repeated $n$-times. Then

$$
\operatorname{diag}\left(\phi\left(a_{i j}\right) e_{11}\right)=W \pi\left(a_{i j}\right) V \text { and }\|W\|=\|V\|=1 .
$$

Thus $\|\phi\|_{c b} \leqq 1$ since $\mathbb{C}^{n^{2}} \rightarrow M_{n^{2}}(\mathbb{C}):\left(\alpha_{j}\right) \rightarrow \operatorname{diag}\left(\alpha_{j} e_{11}\right)$ is a complete isometry.

Let $R$ be the $n \times n^{2}$ matrix with $n$ ones in each row, from $1+(i-1) n$ to $n+(i-1) n$ in the ith row, with all the other elements of $R$ zero; written as $n$ square blocks the $i$ th block in $R$ has a row of ones in the ith row and zeros elsewhere. The $n^{2} \times n$ matrix $T$ is the transpose of $\left(I_{n}, I_{n}, \ldots, I_{n}\right)$, where each $I_{n}$ is the $n \times n$ identity matrix. Then $\left(a_{i j}\right)=\phi^{-1}\left(\phi\left(a_{i j}\right)\right)=R \phi\left(a_{i j}\right) T$, since left multiplication by $R$ drops the elements between $1+(i-1) n$ and in in $\phi\left(a_{i j}\right)$ into the $i$ th row, and post multiplication by $T$ shifts them back to form $\left(a_{i j}\right)$. Now $R R^{*}=n I=T^{*} T$ so $\left\|\phi^{-1}\right\|_{c b} \leqq\|R\| \cdot\|T\|=n$, which completes the proof.

Alternatively Lemma 2.6 may be proved by calculating the completely bounded norms of $\phi$ and $\phi^{-1}$ from the definition of $\|\cdot\|_{c b}$. For $\left\|\phi^{-1}\right\|_{c b}$ an estimate similar to $\left\|\left(\alpha_{i j}\right)\right\| \leqq n \max \left\{\left|\alpha_{i j}\right|: 1 \leqq i, j \leqq n\right\}$ for all $\left(\alpha_{i j}\right) \in M_{n}(\mathbb{C})$ is useful.

The method of proof of 1.1. yields a little more.

Corollary 2.7. An injective von Neumann algebra with separable predual is completely boundedly isomorphic to one, and only one, of the following algebras: $l_{\infty}^{n}(n \in \mathbb{N}), l_{\infty}, B(H)$.

Proof. Note that these representatives of the completely bounded isomorphism classes are not even isomorphic as Banach spaces. Theorem 1.1 shows that only the finite type I von Neumann algebras of bounded degree need be considered. By the standard characterization of these algebras $[5,9,17]$ and Lemma 2.6 , a finite type $I$ von Neumann algebra is completely boundedly isomorphic to a commutative von Neumann algebra with separable predual. A commutative von Neumann algebra with separable predual, is isomorphic to one of the following von Neumann algebras: $l_{\infty}^{n}, l_{\infty}$, $L_{\infty}[0,1], L_{\infty}[0,1] \oplus l_{\infty}^{n}, L_{\infty}[0,1] \oplus l_{\infty}$ (see $[17,1.21$, p. 112]). The last two algebras are completely boundedly isomorphic to $L_{\infty}[0,1]$; this follows from Lemma 2.2 .

Finally we show how to modify the standard Banach space arguments showing that $L^{\infty} \equiv L^{\infty}[0,1]$ is Banach space isomorphic to $l^{\infty}[14]$ to obtain $L^{\infty} c b \cong l^{\infty}$. Clearly $L^{\infty} c b \cong l^{\infty} \oplus X$ for some operator space $X$ by identifying $l^{\infty}$ with a suitable subalgebra of $L^{\infty}$. To apply Lemma 2.2 it only remains to show that $l^{\infty} c b \cong L^{\infty} \oplus Y$.

Choose a sequence $x_{n} \in L^{1} \equiv L^{1}[0,1]$ with

$$
\left\{x_{n}: n \in \mathbb{N}\right\}^{-}=\left\{x \in L_{1}:\|x\|_{1}=1\right\} .
$$

Define $\theta: l^{1} \rightarrow L^{1}$ by $\theta\left(\alpha_{n}\right)=\sum \alpha_{n} x_{n}$. Then $\theta$ is a continuous linear operator onto a dense 
subspace of $L^{1}$. A direct calculation leads to $\left\|\theta^{*}\right\|_{c b} \leqq 1$ on using the definition of complete boundedness and the natural representations of $L^{\infty}$ on $L^{2}$ and of $l^{\infty}$ on $l^{2}$. The inequality $\left\|\theta^{*}-1\right\|_{c b} \leqq 1$ follows from the observation that if $\left(f_{i j}\right) \in M_{n}\left(L^{\infty}\right)$, then

$$
\left\|\left(f_{i j}\right)\right\|=\sup \left\{\left\|\sum_{i, j} f_{i j}(\cdot) \xi_{j} \bar{\eta}_{i}\right\|_{\infty}: \xi, \eta \in \mathbb{C}^{n} \text { with }\|\xi\|=\|\eta\|=1\right\} .
$$

Hence $\theta^{*}$ is a complete isometry from $L^{\infty}$ into $l^{\infty} \subseteq B\left(l^{2}\right)$.

The Wittstock extension theorem for completely bounded operators [12, Theorem 7.2, p. 100] gives an extension $\psi: B\left(l^{2}\right) \rightarrow B\left(L^{2}\right)$ of the completely bounded operator $\theta^{*-1}: \theta^{*}\left(L^{\infty}\right) \rightarrow B\left(L^{2}\right)$ with $\|\psi\|_{c b}=\left\|\theta^{*-1}\right\|_{c b}=1$. Since $L^{\infty}$ is an injective subalgebra of $B\left(L^{2}\right)$, there is a conditional expectation $E$ from $B\left(L^{2}\right)$ onto $L^{\infty}$ with $\|E\|_{c b}=1$. Let $\phi$ from $l^{\infty}$ to $\theta^{*}\left(L^{\infty}\right)$ be defined by $\phi=\left.\theta^{*} \circ E \circ \psi\right|_{\left.\right|^{\infty}}$. Then $\phi$ is a completely bounded projection from $l^{\infty}$ onto $L^{\infty}$ with $\|\phi\|_{c b}=1$. This completes the proof.

Note that $d c b\left(l^{\infty}, L^{\infty}\right) \leqq 2^{8}$.

Note that the equivalence class containing $B(H)$ is of finite $d c b(\cdot, \cdot)$ diameter, but that containing $l_{\infty}$ is of infinite diameter.

We have not tried to obtain the best estimate in $d c b(N, B(H)) \leqq$ constant, and the value given in Theorem 1.1 can be reduced to $2^{12 \frac{1}{2}}$ by exploiting Lemmas 2.9 to 2.12 . The proofs are omitted.

Lemma 2.8 If $A$ is a $C^{*}$-algebra with $A \cong M_{2}(A) \cong M_{3}(A)$, then $d c b(A, A \oplus A) \leqq 12$.

Lemma 2.9. If $N$ is a properly infinite von Neumann algebra, then $\operatorname{dcb}\left(N, N^{n}\right) \leqq n^{1 / 2}$ for all $n \in \mathbb{N}$.

Lemma 2.10. Let $N$ be an injective von Neumann algebra with separable predual that contains no finite type I central summand. Then $N$ is isomorphic to $N \oplus M_{n}(\mathbb{C})$ for all $n \in \mathbb{N}$.

Lemma 2.11. Let $N$ be an injective von Neumann algebra with separable predual. If $N$ is not finite type I of bounded degree, then $N c b \cong N \oplus N$ with $d c b(N, N \oplus N) \leqq 16$.

Lemma 2.12. If $B, N, X, Y$ are operator spaces with

$$
\begin{gathered}
B c b \cong B \oplus B, B c b \cong N \oplus X \\
N c b \cong N \oplus N, N c b \cong B \oplus Y,
\end{gathered}
$$

then $N c b \cong B$ and $d c b(N, B) \leqq \alpha \beta^{2} \gamma \delta^{2}$, where $\alpha, \beta, \gamma, \delta$ are the completely bounded distances in the four isomormpisms, respectively.

The estimate $\operatorname{dcb}(B, N) \leqq 2^{12 \frac{1}{2}}$ now follows with $\alpha \leqq 2^{\frac{1}{2}}, \beta \leqq 2^{4}, \gamma \leqq 2^{4}$ and $\delta \leqq 2^{4}$; this is 
reduced to $3.2^{10 \frac{1}{2}}$ if $N$ has no finite type I central direct summand by Lemma 2.8 . Wassermann and Robertson have independently recently shown that $d c b(N, B(H)) \leqq 2^{8}$, see $[20]$.

Finally note that in proving 1.1 we have essentially proved the following corollary. Note however that there is no bound on $\operatorname{dcb}(N, N \oplus N)$ in general because of finite dimensional type I algebras. For example if $N=M_{n}(\mathbb{C}) \oplus l_{\infty}$, then $d c b(N, N \oplus N)$ tends to infinity as $\boldsymbol{n}$ tends to infinity.

Corollary 2.13. If $N$ is an infinite dimensional injective von Neumann algebra with separable predual, then $N c b \cong N \oplus N$.

Szankowski's result that $B(H)$ does not have the approximation property [15] combined wth 1.1. gives the following corollary.

Corollary 2.14. If $N$ is an injective von Neumann algebra with separable predual not finite type I of bounded degree, then $N$ does not have the approximation property.

\section{Type $\mathrm{II}_{1}$ algebras}

If $x \in N^{\infty}$, regard $x=\left(x_{1}, x_{2}, \ldots\right)$. Let $L$ denote the left shift on $N^{\infty}$, that is, $L x=$ $\left(x_{2}, x_{3}, \ldots\right)$.

Lemma 3.1 Let $N$ be a von Neumann algebra. If $\phi$ is a normal *-monomorphism from $N \oplus N$ into $N$, then there is a normal *-monomorphism $\theta$ from $N^{\infty}$ into $N$ such that $\theta(x)=\phi\left(x_{1} \oplus \theta(L x)\right)$ for all $x \in N^{\infty}$, where $x=\left(x_{j}\right)$ and $L$ is the left shift on $N^{\infty}$.

Proof. Let $e_{n}=(1, \ldots, 1,0, \ldots)$ be in $N^{\infty}$ with $n$ ones in the first $n$ places and zeros thereafter; then $N^{n}$ is isomorphic to $e_{n} N^{\infty}$. Define $\theta_{j}$ inductively on $N^{\infty}$ by

$$
\theta_{1}(x)=\phi\left(x_{1} \oplus 0\right) \quad \text { and } \quad \theta_{n}(x)=\phi\left(x_{1} \oplus \phi\left(x_{2} \oplus \phi\left(\ldots \oplus \phi\left(x_{n} \oplus 0\right) \ldots\right)\right)\right) .
$$

The last equation is equivalent to

$$
\theta_{n}(x)=\phi\left(x_{1} \oplus \theta_{n-1}(L x)\right)
$$

Then each $\theta_{n}$ is a normal *-homomorphism from $N^{\infty}$ into $N$, and $\theta_{n}$ is a monomorphism when restricted to $N^{n}$. Further

$$
\left.\theta_{n+k}\right|_{N^{n}}=\left.\theta_{n}\right|_{N^{n}}
$$

for all $n, k \geqq 1$. Hence $\theta_{n}\left(N^{n}\right)$ is an increasing sequence of weakly closed *-subalgebras of $N$; let $W$ be the weak closure of $W_{0}=\cup \theta_{n}\left(N^{n}\right)$. The boundedness of the sequence $\left(\theta_{n}(x): n \in \mathbb{N}\right)$ implies that if LIM is a Banach limit on $l^{\infty}$, then $\theta(x)=\operatorname{LIM} \theta_{n}(x)$ exists in the weak topology in $N$ for each $x \in N^{\infty}$. The equation

$$
\theta_{n+k}\left(e_{n} x\right)=\theta_{n+k}\left(e_{n}\right) \theta_{n+k}(x)=\theta_{n}\left(e_{n} x\right)=\theta_{n}(x)
$$


$(n, k \geqq 1)$ ensures that

$$
\theta\left(e_{n} x\right)=\theta_{n}\left(e_{n}\right) \theta(x)=\theta_{n}(x)
$$

for all $x \in N^{\infty}$. Also

$$
\theta_{n}\left(e_{n}\right)=\theta_{n+1}\left(e_{n} e_{n+1}\right)=\theta_{n}\left(e_{n}\right) \theta_{n+1}\left(e_{n+1}\right) \leqq \theta_{n+1}\left(e_{n+1}\right),
$$

so $\theta_{n}\left(e_{n}\right)$ is an increasing sequence of projections in $W$, which converges strongly to the identity $\theta(1)$ of $W$ because $\theta_{n}\left(e_{n}\right) \theta(x)=\theta_{n}(x)(n \in \mathbb{N})$. The last equation then implies that $\theta_{n}(x)$ converges strongly to $\theta(x)$ for all $x \in N^{\infty}$. From this and each $\theta_{n}$ being a *-homomorphism from $N^{\infty}$ into $N$ it follows that $\theta$ is a *-homomorphism. The inequality

$$
\begin{aligned}
|\langle\theta(x) \xi, \eta\rangle| & \leqq\left\|\left(\theta(x)-\theta_{n}(x)\right) \xi\right\| \cdot\|\eta\|+\left|\left\langle\theta_{n}(x) \xi, \eta\right\rangle\right| \\
& \leqq \|\left(\theta(1)-\theta_{n}\left(e_{n}\right) \xi \xi\|\cdot\| x\|\cdot\| \eta \|+\left|\left\langle\theta_{n}(x) \xi, \eta\right\rangle\right|\right.
\end{aligned}
$$

for $x \in N^{\infty}$ and $\xi, \eta \in H$, shows that $\theta$ is a normal operator on the closed unit sphere $\left(N^{\infty}\right)_{1}$ of $N^{\infty}$ since each $\theta_{n}$ is normal. Since a linear functional weakly continuous on the unit sphere of a von Neumann algebra is weakly continuous on the algebra $[9,7.4 .5, p$. 483], $\theta$ is a normal operator. If $\theta(x)=0$, then $\theta_{n}\left(e_{n} x\right)=\theta_{n}\left(e_{n}\right) \theta(x)=0$ so that $e_{n} x=0$ because $\theta_{n}$ is a monomorphism on $N^{n}$; so $x=0$ and $\theta$ is a monomorphism. The equation satisfied by $\theta$ and $\phi$ follows from the definitions of $\theta_{n}$ and $\theta$, and the strong convergence of $\theta_{n}(x)$ to $\theta(x)$.

Lemma 3.2. Let $N$ be a type $I I_{1}$ von Neumann algebra. If there is a normal *-homomorphism from $N \oplus N$ into $N$, then $N \sigma c b \cong N^{\infty}$ by an involution preserving operator; further $d \sigma c b\left(N, N^{\infty}\right) \leqq 16$.

Proof. By Lemma 3.1 there is a normal *-monomorphism $\theta$ from $N^{\infty}$ onto the weakly closed subalgebra $\theta\left(N^{\infty}\right)$ of $N$. Let $E$ be a conditional expectation from $N$ onto $\theta\left(N^{\infty}\right)$; note that $E$ is a completely positive operator preserving the identity. Then $N \sigma c b \cong N^{\infty} \oplus X$, where $X=\operatorname{Ker} E$, and the completely bounded distance between these spaces is bounded by 4 . Hence

$$
N^{\infty} \oplus N \sigma c b \cong N^{\infty} \oplus N^{\infty} \oplus X \sigma c b \cong N^{\infty} \oplus X \sigma c b \cong N
$$

with the involution preserved. Clearly $d \sigma c b\left(N, N^{\infty}\right) \leqq 16$.

3.3. Proof of Theorem 1.2. Lemmas 3.1 and 3.2. together with the conditional expectation from a type $\mathrm{II}_{1}$ von Neumann algebra onto a weakly closed *-algebra (not necessarily containing the identity of the whole algebra) give the hypotheses of Lemma 2.2 for completely bounded normal isomorphisms. 


\section{REFERENCES}

1. E. Christensen, Derivations and their relation to perturbations of operator algebras, Proc. Sympos. Pure Math. 38 (1982) 2, 261-273.

2. E. Christensen, Perturbations of operator algebras II, Indiana Univ. Math. J. 26 (1977), 891-904.

3. A. Connes, Classification of injective factors, Ann. of Math. 104 (1976), 73-116.

4. M. Cowling and U. HaAgerup, Completely bounded multipliers of the Fourier algebra of a simple Lie group of real rank one, preprint.

5. J. Dixmıer, Les algèbres d'operateurs dans l'espace Hilbertien, 2nd edition (Gauthier-Villars, Paris, 1969).

6. U. HaAGerup, Group $C^{*}$-algebras without the completely bounded approximation property, in preparation.

7. B. E. Johnson, Perturbations of Banach algebras, Proc. London Math. Soc. 34 (1977), $439-458$.

8. R. V. KADISON, Isometries of operator algebras, Ann. of Math. 54 (1951), 325-338.

9. R. V. Kadison and J. R. Ringrose, Fundamentals of the theory of operator algebras, Vol. 2 (Academic Press, New York, 1986).

10. J. Lindenstrauss and L. TzafriRı, Classical Banach Spaces, Sequence Spaces (Ergebnisse 92, Springer Verlag, 1977).

11. F. J. Murray and J. von Neumann, On rings of operators, Ann. of Math. 37 (1936), $116-229$.

12. V. I. Paulsen, Completely bounded maps and dilations (Pitman Research Notes in Mathematics, Longman, London 1986).

13. Z. Ruan, Subspaces of $C^{*}$-albegras, J. Funct. Anal. 76 (1988), 217-230.

14. H. P. Rosenthal, On injective Banach spaces and the spaces $L^{\infty}(\mu)$ for finite measures $\mu$, Acta Math. 124 (1970), 205-248.

15. Z. Semadeni, Banach spaces of continuous functions (PWN, Warsaw, 1971).

16. A. Szankowsk, $B(H)$ does not have the approximation property, Acta Math. 147 (1981), 89-108.

17. M. TAKESAK!, Theory of operator algebras I (Springer Verlag, Berlin, 1979).

18. N. Tomczack-Jaegerman, Buriach-Mazur distances... (Pitman, London, 1988).

19. S. Wassermann, On tensor products of certain group $C^{*}$-algebras, J. Funct. Anal. 23 (1976), 239-254.

\section{Reference added in proof.}

20. A. G. Robertson and S. Wassermann, Completely bounded isomorphisms of injective operator systems, Bull. London Math. Soc. 21 (1989), to appear.

Matematisk Institut

KOBENHAVN UNIVERSITET

UNIVERSITETSPARKEN 5

2100 KoBENHAVN

DENMARK
Department of Mathematics UNIVERSITY OF EDINBURGH James Clerk Maxwell Building KING'S BUILDINGS EDINBURGH EH9 3JZ 